\title{
The grammar of approximating number pairs
}

\author{
KIMMo ERIKSSON \\ Mälardalen University, Västerås, Sweden \\ AND \\ DReW H. BaILeY AND DaVID C. GEARY \\ University of Missouri, Columbia, Missouri
}

\begin{abstract}
In the present article, we studied approximating pairs of numbers $(a, b)$ that were used to estimate quantity in a single phrase ("two, three years ago"). Pollmann and Jansen (1996) found that only a few of the many possible pairs are actually used, suggesting an interaction between the ways in which people estimate quantity and their use of quantitative phrases in colloquial speech. They proposed a set of rules that describe which approximating pairs are used in Dutch phrases. We revisited this issue in an analysis of Swedish and American language corpora and in a series of three experiments in which Swedish and American adults rated the acceptability of various approximating pairs and created approximating pairs of their own in response to various estimation tasks. We found evidence for Pollmann and Jansen's rules in both Swedish and English phrases, but we also identified additional rules and substantial individual and cross-language variation. We will discuss implications for the origin of this loose "grammar" of approximating pairs.
\end{abstract}

It is likely that humans have some form of inherent system for representing quantity - specifically, for representing exact quantities up to three and for making reasonable estimates for larger quantities (see, e.g., Dehaene, 1997; Gallistel \& Gelman, 1992). Number words (e.g., "five") and other quantitative words and phrases (e.g., "more than") are mapped onto these inherent representations in ways that allow people to communicate about quantity. Much is known about children's mapping of number words onto cognitive representations of specific quantities (Fuson, 1988) and about how children estimate on mathematical tasks, such as a number line (Siegler \& Opfer, 2003). Much less is known about how quantitative phrases are used in colloquial speech and whether there are discernible patterns in these phrases that reflect constraints on how people inherently represent quantities, whether they are based on the structure of the school-taught base10 number system and other cultural systems (e.g., time), or whether they are some combination of constraint and cultural conventions.

Dehaene and Mehler's (1992) cross-linguistic study of people's number use uncovered an inverse relationship between the magnitude of numbers and the frequency of usage, with local peaks at numbers such as $10,12,15$, 20,50 , and 100 . The same basic pattern was found for all seven languages assessed in this study. Dehaene and Mehler concluded that the preference for small numbers may have been due to the more precise underlying quantitative representations, whereas the prevalence of "reference nu- merals," such as 10 and 50, reflects use of our less precise approximations for larger quantities. Reference numerals may provide an anchor for mapping numerals and number words onto the underlying approximate representational system and for guiding a variety of daily activities in modern cultures. When searching for a street address at 1615 , an understanding of the quantitative sequence of $100,200, \ldots 1000$ allows people to scan for the correct block and to focus attention on individual addresses once the 1600 block is reached. At the same time, studies of traditional cultures without formal education indicate a much more limited range of number words that likely map onto the precise representations for small quantities (Chagnon, 1997; Pica, Lemer, Izard, \& Dehaene, 2004), although number words for quantities beyond this range are sometimes found (Saxe, 1981).

The cross-cultural pattern suggests that the development of representations for quantity - number words, Arabic numerals - results from a combination of a social use for these words and numerals and an ability to map these onto precise or approximate representations of quantity. Dehaene and Mehler's (1992) fascinating finding that the same reference numerals $(10,12,15,20,50$, and 100) appear in many languages and modern cultures suggests a regularity in either the culturally derived number systems that map onto the approximate range or in how people intuitively use the approximate system for developing number systems. Many reference numerals are multiples of powers of 10 (which may follow from the total number 
of fingers on our two hands) and divisors of 60 (Dehaene $\&$ Mehler, 1992). The latter is a useful reference numeral (e.g., $60 \mathrm{sec}=1 \mathrm{~min}$ ) because it has many divisors (e.g., $2,3,10,12,15)$.

Whatever their source, reference numerals provide a means for communication of an approximate magnitude ("about 20 years ago"). People also use pairs of reference numerals, or an approximating pair, such as "20, 25 years ago," for more precise communication of the degree of uncertainty involved. The use of pairs of numerals to estimate and to communicate a range of quantities has, under various names, been the subject of several previous studies (Channel, 1980; Langendoen, 2006; Pollmann \& Jansen, 1996; Sigurd, 1988). We do not fully understand what makes an acceptable approximating pair, but we do know that not every possible pair is an acceptable expression (e.g., no one would say "7, 19 years ago"). The latter suggests that there may be constraints, a "grammar" of approximating pairs, on the combinations of numbers that people use to convey approximate ranges of quantity. Sigurd argued that this "grammar" is simply a linguistic convention, with no underlying constraints tied to how people represent approximate magnitude. In the only previous empirical study on the topic, Pollmann and Jansen argued that the grammar of approximating pairs is more fundamentally determined: "No special training is needed . . . People simply seem to know what differences are allowed between members of approximative expressions" (p. 230). As in Dehaene and Mehler's (1992) analysis, Pollmann and Jansen sought to determine which approximating pairs are preferred and why.

\section{Pollmann and Jansen's (1996) Principles}

Pollmann and Jansen (1996) analyzed two large Dutch databases of written and spoken language. They identified a total of 187 instances of approximating pairs $(a, b)$ in these corpora and, on the basis of an analysis of these pairs and their difference $(d=b-a)$, identified a set of necessary conditions for an approximating pair to be acceptable in Dutch phrases. These conditions were formulated in terms of arithmetic sequences, but for simplicity, we reformulated them directly in terms of the difference:

- The second number rule: The second number must be larger than the first; $d>0$.

- The divisor rule: The difference must be a divisor of the second number; $d \mid b$ (in which case, by mathematical necessity, it is also a divisor of the smaller number).

- The $5 \%$ rule: The difference must be at least $5 \%$ of the second number; $d \geq b / 20$.

- The favorite numbers rule: The difference must equal $1,2,2.5$, or 5 after division by a suitable power of $10 ; d \in F(10)$, where $F(10)$ is defined as the set $\left\{m \times 10^{n} \mid n\right.$ integer, $m=1,2,2.5$, or 5$\}$.

As an example, consider the pair $(4,6)$. The second number is larger than the first; the difference of 2 is a divisor of both 4 and 6; 2 is $>6 / 20$; and 2 is in the set $F(10)$ - that is, $2 \times 10^{0}$. The pair $(20,30)$ provides another example. The second number is larger than the first; 10 is a divisor of both 20 and 30 and is $>30 / 20$. The favorite numbers rule is satisfied because $10=1 \times 10^{1}$, or, stated differently, the difference of 10 is in the set of $1,2,2.5$, or 5 , after division by $10^{1}$.

Pollmann and Jansen's (1996) main interest was with the fourth rule, which for a general base $B$ of the number system, states that there exists a set $F(B)$ of favorite numbers that humans will find acceptable in pairwise approximations; specifically, this set will include the powers of the base and their doubles, halves, and halves of halves. Thus, in our base-10 number system, the set $F(10)$ of favorite numbers contains $1,2,2.5$, and 5 , and all possible shifts by a power of 10 (e.g., 10, 50). United States coins and other modern currencies, for instance, follow from this rule: Pennies, nickels, dimes, quarters, and half dollars all are consistent with the favorite numbers rule. Starting from the base of one dollar, a dime represents a shift of 10, a nickel is half a dime, and a quarter is half of a half dollar. According to Pollmann and Jansen,

The principle of favorite numbers resembles the modules of Universal Grammar in the Chomskian sense. It may be a cognitive module comparable with Government theory, Case theory and the like (Chomsky, 1981, p. 135). Each of these theories consists of a set of principles that function as a kind of filter that discriminates between "possible" and "impossible" expressions (p. 232).

The explanation offered for the origin of such universal principles is the existence of the analog magnitude code for representing approximate quantities (Dehaene, 1992) and the particular simplicity of the two operations of halving and doubling.

\section{Unresolved Issues}

Assuming the existence of constraints underlying the four Pollmann and Jansen (1996) rules, the support for the aforementioned explanation of its origin is not compelling. To be sure, humans in modern cultures do learn to map culturally important number words, Arabic numerals, and other symbols of magnitude onto the inherent approximate representational system (Geary, 2006; Siegler \& Opfer, 2003; Spelke, 2000), but whether the patterns identified by Pollmann and Jansen (1996) reflect inherent biases in how humans parse quantities mapped onto this system or reflect the structure of the culturally taught base-10 system is not known. More fundamentally, data do not clearly support the claim that approximating pairs are used "only if" the four rules are met (Pollmann \& Jansen, 1996, p. 224). In the Dutch corpus data, every condition was violated at least once: Pollmann and Jansen reported occurrences of $(5,4)$, which breaks the second number rule; $(1,4)$, which breaks the divisor rule; $(1,918$, $1,920)$, which breaks the $5 \%$ rule; and $(12,15)$, which breaks the favorite numbers rule. It is not known whether these violations are errors, are special cases that appear only in particular contexts, or reflect rules that were not identified by Pollmann and Jansen. 
Moreover, a detailed look at Pollmann and Jansen's (1996) data suggests that the favorite numbers rule may be an oversimplification. Eighty percent of their identified pairs had a difference of 1 (times some power of 10 ), less than $20 \%$ of the pairs had a difference of 2 or 5 (times some power of 10), and differences of 2.5 were totally absent. This indicates that some of the proposed "favorite numbers" are more favored than others. In this context, it is noteworthy that Langendoen (2006), although without any supporting data, proposed a set of principles that are equivalent to the second number rule, the difference rule, and the favorite numbers rule - with the exception that Langendoen excluded 2.5 as an acceptable difference.

Finally, Pollmann and Jansen's (1996) corpus analysis has several important limitations. The sample of approximating pairs that one obtains from such corpora is small and biased in unknown ways. Although the corpora document usage of expressions, they provide no information on whether people find this usage acceptable or not, or whether they find other combinations that are not found in the corpora acceptable.

\section{The Present Experiments and Research Questions}

To achieve a more complete understanding of the "grammar" of approximating pairs and its cognitive underpinnings, we designed a series of experiments in Sweden and the U.S. to assess the generalizability and robustness of Pollmann and Jansen's (1996) rules. Specifically, we asked the following five research questions.

1. Are the same approximating pairs used, and found acceptable, in Swedish and English?

2 . Is the acceptability of approximating pairs dichotomous (i.e., pairs are either wholly acceptable or wholly unacceptable), as was implicitly assumed by Pollmann and Jansen?

3. To what extent does Swedish and English usage of approximating pairs follow the rules proposed by Pollmann and Jansen, and are there other crosslanguage rules that can be identified?

4. Are there individual differences in the application of these rules?

\section{Are the rules for usage of approximating pairs con-} text sensitive?

We used several methods to address these questions. In the first experiment, we analyzed Swedish and English language corpora to identify approximating pairs in use (Question 1); we also analyzed how frequently different pairs were used to address Question 2, and whether the frequently used pairs were the same as the pairs that conformed to the rules identified by Pollmann and Jansen in the Dutch corpora to address Question 3.

The remaining studies used questionnaires. In Experiment 2A, we asked Swedish and American college stu- dents to create approximating pairs in given contexts. Since, as we discussed previously, it is not necessarily the case that people accept exactly the same approximating pairs that others use, we also asked the same participants to judge the acceptability of various approximating pairs (Experiment 2B). These experiments allowed us to revisit Questions 1-3 with different kinds of data.

To anticipate, we found in these experiments that Pollmann and Jansen's (1996) rules have considerable explanatory power, but that they do not explain the entire set of approximating pairs or the conditions of their usage and acceptability; indeed, we found features of the data that suggest several new rules. In Experiment 3, we asked Swedish and American participants to rate the familiarity of a large number of approximating pairs; we subjected this data set to extensive multilevel analysis to test the validity of our newly suggested rules as well as those of Pollmann and Jansen. This analysis allowed us to assess not only national differences in the application of these rules, but also individual differences (Question 4).

The final question about the context sensitivity of the rules comes from a suggestion by Pollmann and Jansen (1996) that other approximating pairs may be used when the context of the approximation is measurement in a unit that is implicitly counted in some other base than 10 . For instance, days are counted in base 7 when grouped into weeks; similarly, hours are counted in base 24 when grouped into days, whereas minutes are counted in base 60 when grouped into hours. In Experiment 4, we addressed this question by asking Swedish students to judge the acceptability of pairs when we manipulated context.

\section{EXPERIMENT 1}

We complemented Pollmann and Jansen's (1996) study of the Dutch corpora with a similar analysis of two other corpora: the Swedish PAROLE corpus at Språkbanken, Göteborg, consisting of around 19 million running-text words, and the 20 times larger Corpus of Contemporary American English (Davies, 2008), which contains 385 million words. Both data sets are freely available (http://spraakbanken .gu.se/parole/ and www.americancorpus.org).

\section{Method}

We searched both databases for all possible approximating pairs involving numbers between 2 and 20. To assess the favorite numbers rule, we also searched for four specific pairs, two that conformed to the rule $(25,50 ; 50,75)$ and two that did not $(30,60 ; 60,90)$. The difference for the conforming pairs was 25 , which conformed to the rule because $25=2.5 \times 10^{1} ; 2.5$ is in the identified set of $1,2,2.5$, and 5 (Pollmann \& Jansen, 1996). The nonconforming pairs had the same ratios (1:2 and 2:3) as the conforming one, but were not consistent with the favorite numbers rule.

\section{Results}

Table 1 shows the frequencies of the approximating pairs in the Swedish and English language corpora. Due to the very large American corpus, the table reports only pairs with at least 10 occurrences. The frequency counts from the two data sets are highly correlated $(r=.93, p<$ 
Table 1

Frequencies of Number Pairs in the Swedish PAROLE Corpus and the Corpus of Contemporary American English (COCA)

\begin{tabular}{|c|c|c|c|c|c|}
\hline Pair & $\begin{array}{l}\text { Swedish } \\
\text { Frequency }\end{array}$ & $\begin{array}{l}\text { English } \\
\text { Frequency }\end{array}$ & Pair & $\begin{array}{l}\text { Swedish } \\
\text { Frequency }\end{array}$ & $\begin{array}{c}\text { English } \\
\text { Frequency }\end{array}$ \\
\hline 2,3 & 74 & 8,361 & 12,13 & 2 & 248 \\
\hline 2,4 & 0 & 60 & 12,14 & 1 & 62 \\
\hline 2,5 & 0 & 24 & 12,15 & 1 & 60 \\
\hline 3,4 & 90 & 5,750 & 12,18 & 0 & 11 \\
\hline 3,5 & 0 & 67 & 13,14 & 0 & 133 \\
\hline 3,6 & 1 & 23 & 14,15 & 0 & 145 \\
\hline 4,5 & 68 & 3,596 & 14,16 & 0 & 12 \\
\hline 4,6 & 0 & 61 & 15,16 & 2 & 157 \\
\hline 4,8 & 0 & 34 & 15,20 & 3 & 527 \\
\hline 5,6 & 58 & 2,715 & 16,17 & 0 & 98 \\
\hline 5,7 & 0 & 55 & 16,18 & 0 & 14 \\
\hline 5,8 & 0 & 11 & 17,18 & 0 & 104 \\
\hline 5,10 & 0 & 340 & 18,19 & 0 & 89 \\
\hline 6,7 & 30 & 1,490 & 18,20 & 0 & 41 \\
\hline 6,8 & 2 & 343 & 19,20 & 0 & 59 \\
\hline 6,9 & 0 & 23 & & & \\
\hline 6,12 & 0 & 10 & & & \\
\hline 7,8 & 27 & 1,070 & 25,50 & 0 & 12 \\
\hline 7,10 & 0 & 10 & 30,60 & 0 & 17 \\
\hline 8,9 & 6 & 688 & 50,75 & 0 & 15 \\
\hline 8,10 & 4 & 212 & 60,90 & 0 & 12 \\
\hline 9,10 & 2 & 272 & & & \\
\hline 10,11 & 2 & 305 & & & \\
\hline 10,12 & 9 & 483 & & & \\
\hline 10,15 & 12 & 765 & & & \\
\hline 10,20 & 5 & 382 & & & \\
\hline
\end{tabular}

Note-The frequencies refer to pairs expressed using either digits ("2 or 3") or words ("two or three"). Frequencies are presented for all pairs in which both numbers are between 2 and 20, and in which there is at least 1 occurrence in PAROLE or at least 10 occurrences in COCA. In addition, frequencies are presented for four selected pairs with larger numbers.

.001 ), indicating that the usage of approximating pairs is very similar in Sweden and America.

Consistent with Pollmann and Jansen's (1996) findings for Dutch, all but one of the Swedish approximating pairs was consistent with their four rules. The exception was the pair $(12,15)$, for which the difference of $3 \mathrm{did}$ not satisfy the favorite numbers rule. We also note that many approximating pairs that conform to the Pollmann and Jansen rules are not found in the Swedish corpus; for instance, the pairs $(2,4)$ and $(18,20)$ are absent, although they are acceptable pairs on the basis of the four rules.

The American corpus is much larger; thus, it is not surprising that it contains a larger variety of approximating pairs. Unlike in the Swedish corpus, all of the approximating pairs between 2 and 20 that conform to Pollmann and Jansen's (1996) rules are present in the American corpus, but there are also several pairs that do not conform to these rules. For instance, the three pairs $(3,5),(5,7)$, and $(7,10)$ violate the divisor rule. Similarly, the six pairs $(4,8),(6,9),(6,12),(7,10),(12,15)$, and $(12,18)$ violate the favorite numbers rule.

Finally, Table 1 shows that the conforming pairs of $(25$, $50)$ and $(50,75)$ never occur in the Swedish corpus and only rarely in the American one. The corresponding frequencies are nearly identical to those of the nonconforming pairs of $(30,60)$ and $(60,90)$.

\section{Discussion}

Recall that the first three research questions listed in the introduction asked whether the same approximating pairs are used in Swedish and American English, whether acceptability is dichotomous or whether there is a gray area of more or less acceptable pairs, and whether pairs in use follow the rules that were proposed by Pollmann and Jansen (1996). We found that the frequency of occurrence of approximating pairs in both Swedish and English is indeed highly similar to that found in Dutch, and that most of these pairs conform to Pollmann and Jansen's four rules. Nevertheless, we also found notable exceptions. First, there are pairs in both Swedish and English that do not conform to these rules (e.g., 12, 15) but that are used as frequently as some pairs that do conform to them (e.g., 12, 14). Second, there are pairs that conform to the rules that are rarely, if ever, used, and this variation in usage shows some definite patterns. For instance, pairs with smaller numbers occur more frequently than do those with larger ones, as was previously found by Dehaene and Mehler (1992) for single numerals and number words. Overall, the variation in frequencies in Table 1 is huge, strongly suggesting that not all acceptable pairs are equally acceptable.

In the following experiments, we made a more thorough exploration of which factors account for the variation in the usage of different approximating pairs.

\section{EXPERIMENT 2A}

In Experiment 1, we found that a highly similar set of approximating number pairs were used in Swedish and English. In Experiment 2A, we examined whether the same pairs emerged when undergraduates were asked to provide approximating pairs to estimate a quantitative range.

\section{Method and Procedures}

A 12-item questionnaire was administered to 105 U.S. undergraduates at the University of Missouri and to 72 Swedish undergraduates at Mälardalen University. The questionnaire was administered in a group setting and required about 10 to $15 \mathrm{~min}$ to complete. Each of the items asked participants to create approximating pairs that estimated a range of values. The content of the questions was based on general knowledge, but was modified for each society (e.g., pounds in the U.S., kilograms in Sweden). As an illustration, two of the items read as follows: "How heavy is George W. Bush, do you think? Hm. I'd say he weighs ____ pounds. How many days would it take for you to go by bike from Columbia, MO to Columbia, SC? With a good bike, I think it would take me ___ days."

\section{Results and Discussion}

A total of 156 individual numbers were used in the approximating pairs, and Figure 1 shows the absolute frequencies of the 20 most commonly used numbers; these 20 numbers constitute about $75 \%$ of the entire data set. The correlation between frequencies from the U.S. and Swedish samples is high $(r=.85, p<.001)$, despite the aforementioned differences in units, replicating the finding in Experiment 1.

As was found by Dehaene and Mehler (1992), for both samples, smaller numbers tended to occur more 


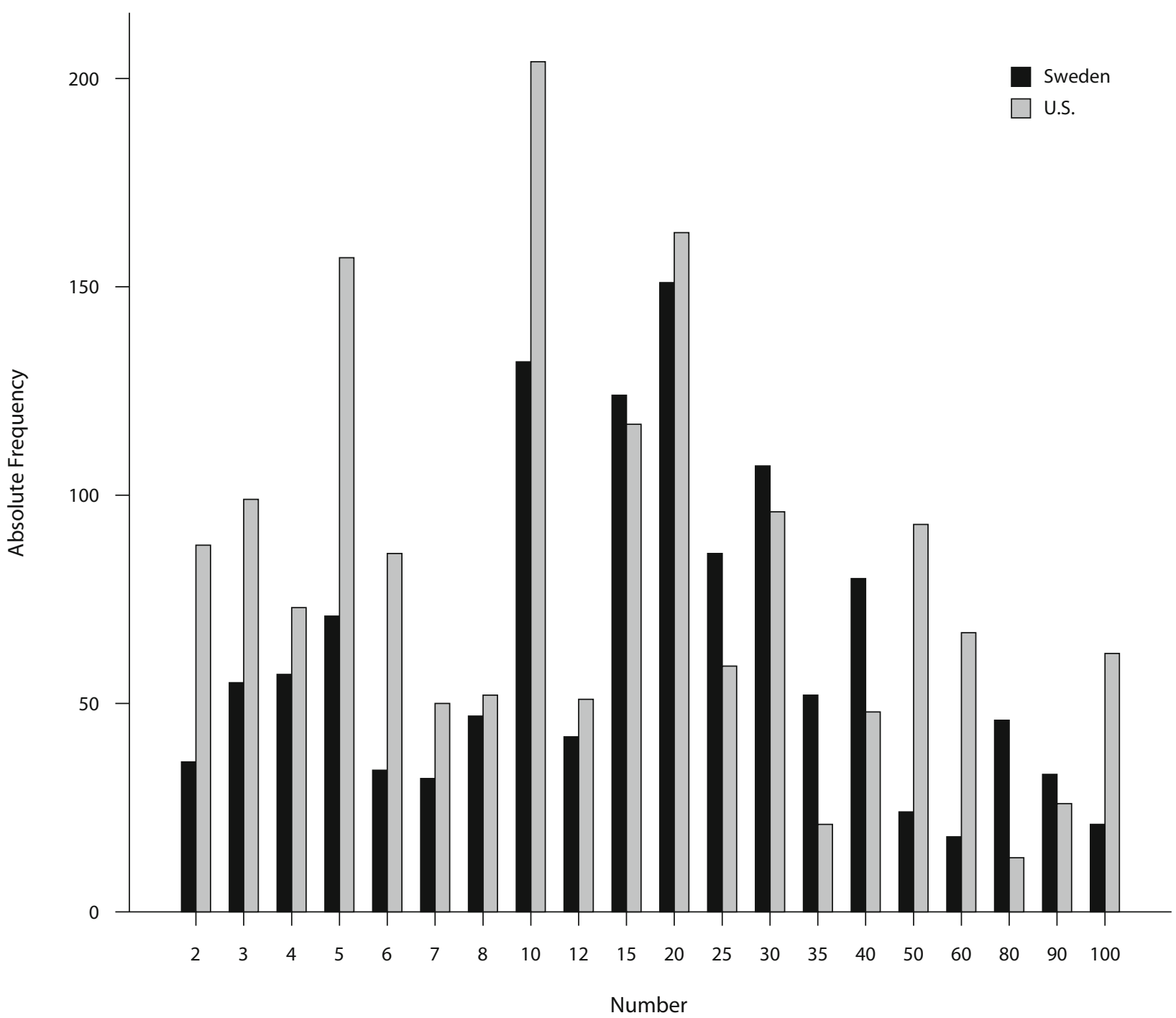

Figure 1. Absolute frequencies of the 20 most common numbers used in the 1,924 approximating pairs created by participants in Experiment 2A.

frequently than larger ones, and with the same local peaks at $10,12,15,20,50$, and 100 . In addition to these local peaks, a few additional peaks emerged for both the Swedish and the U.S. students (e.g., at 5 and 30). Indeed, despite the bias for smaller numbers, as many as 13 of the 20 most frequent numbers were multiples of 5 (Figure 1).

Following the study of Pollmann and Jansen (1996), we also computed the difference $(b-a)$ of each approximating pair $(a, b)$ generated by our participants. Table 2 reports frequencies of the 20 most common differences, constituting almost $99 \%$ of the data set. Of the corresponding 1,924 generated pairs, $83 \%$ conformed to Pollmann and Jansen's four rules. The remaining 17\% violated at least one rule. A separate assessment of the necessity of each of these conditions indicated that the second number rule was violated by $1.6 \%$ of the approximating pairs, and the divisor, $5 \%$, and favorite numbers rules were violated by $5.5 \%, 8.7 \%$, and $5.9 \%$ of the pairs, respectively.

As can be seen in Table 2 and Table 3, Pollmann and Jansen's (1996) predicted favorite number of 2.5including powers of 10 (e.g., 2.5, 5; 25, 50; 250, 500)was rarely generated by Swedish or U.S. participants. The pattern strongly suggests that 2.5 should be removed from the set of favorite numbers that is associated with the favorite numbers rule. In fact, although 3 and 4 are not among the proposed favorite numbers, they are both used significantly more frequently than $2.5(p<.001$ in both cases, binomial test).

To summarize, these questionnaire results replicate the findings from our corpus analysis regarding Research Questions 1 and 3: The usage of approximating pairs is similar in Swedish and English, and it follows the rules of Pollmann and Jansen (1996) with some notable exceptions; in particular, 2.5 seems not to be a true favorite number. 
Table 2

Distribution of the 20 Most Common Differences in the Set of 1,924 Approximating Pairs Created in Experiment 2A

\begin{tabular}{cccc}
\hline Difference & Frequency (\%) & Difference & Frequency $(\%)$ \\
\hline 5 & 33.7 & 30 & 0.4 \\
1 & 31.6 & 100 & 0.4 \\
10 & 16.5 & 6 & 0.3 \\
2 & 7.8 & -10 & 0.3 \\
3 & 1.6 & 15 & 0.3 \\
4 & 1.5 & 25 & 0.3 \\
50 & 1.2 & -2 & 0.2 \\
20 & 1.0 & 12 & 0.2 \\
-5 & 0.7 & -3 & 0.1 \\
7 & 0.4 & -1 & 0.1 \\
\hline
\end{tabular}

Note-Differences are presented in the order of decreasing frequency. Not shown are 23 differences that occurred with a frequency of less than $0.1 \%$, constituting the remaining $1.5 \%$ of the data set.

\section{EXPERIMENT 2B}

The focus of Experiments 1 and $2 \mathrm{~A}$ was on the usage and generation of approximating pairs used to estimate a quantitative range. In Experiment 2B, we moved on to assess participants' ratings of the acceptability of presented approximating pairs. This is analogous to linguistic analyses of the grammatical acceptability of different utterances (see Luka \& Barsalou, 2005).

\section{Method}

A 16-item questionnaire was administered to the same participants as in Experiment 2A; 104 U.S. participants and 70 Swedish participants completed the questionnaire. The items involved the judgment of acceptability of approximating pairs. Participants were presented with phrases that included various approximating pairs - for example, "How long ago was Gandhi murdered? Fifty, sixty years ago?" Participants were asked to judge how natural they thought the approximating expression would sound in speech, on a 1 (completely unnatural) to 5 (completely natural) scale. Of the 16 phrases, 7 included approximating pairs that conformed to Pollmann and Jansen's (1996) four rules, and 9 included pairs that violated at least one of the rules.

Table 3

All Positive Pollmann and Jansen (1996) Pairs up to Scaling With a Suitable Power of 10

\begin{tabular}{cccc}
\hline Difference 1 & Difference 2 & Difference 2.5 & Difference 5 \\
\hline 1,2 & 2,4 & $2.5,5$ & 5,10 \\
2,3 & 4,6 & $5,7.5$ & 10,15 \\
3,4 & 6,8 & $7.5,10$ & 15,20 \\
4,5 & 8,10 & $10,12.5$ & 20,25 \\
5,6 & 10,12 & $12.5,15$ & 25,30 \\
6,7 & 12,14 & $15,17.5$ & 30,35 \\
7,8 & 14,16 & $17.5,20$ & 35,40 \\
8,9 & 16,18 & $20,22.5$ & 40,45 \\
9,10 & 18,20 & $22.5,25$ & 45,50 \\
10,11 & 20,22 & $25,27.5$ & 50,55 \\
11,12 & 22,24 & $27.5,30.5$ & 55,60 \\
12,13 & 24,26 & $30,32.5$ & 60,65 \\
13,14 & 26,28 & $32.5,35$ & 65,70 \\
14,15 & 28,30 & $35,37.5$ & 70,75 \\
15,16 & 30,32 & $37.5,40$ & 75,80 \\
16,17 & 32,34 & $40,42.5$ & 80,85 \\
17,18 & 34,36 & $42.5,45$ & 85,90 \\
18,19 & 36,38 & $45,47.5$ & 90,95 \\
19,20 & 38,40 & $47.5,50$ & 95,100 \\
\hline
\end{tabular}

Note-Italicized pairs did not occur in the data set of Experiment 2A.

\section{Results and Discussion}

The mean judgments for all approximating pairs are shown in Table 4; pairs preceded by "+" conform to Pollmann and Jansen's (1996) four rules. When rating the acceptability of 16 expressions on a 1-5 scale, only 4 out of 174 participants used only two rating steps (namely, 1 and 5), whereas most participants made use of the entire $1-5$ range. The mean number of rating steps used was 4.56 $(S D=0.76)$, which is significantly larger than $2(p<$ $.001)$. We conclude that acceptability is most appropriately modeled as a continuous, rather than dichotomous, variable (Question 2).

Revisiting Question 1, we find the correlation between mean judgments of the U.S. and Swedish sample to be very high $(r=.85, p<.001)$, indicating a substantial overlap in the acceptability ratings of approximating pairs. However, we observe two systematic differences in Table 4 between the samples, both indicating that multiples of 5 are relatively more favored in American English than in Swedish. Pairs that include at least one number that is not a multiple of $5(57,60 ; 58,62 ; 58,60 ; 60,62 ; 20,23 ; 18$, $22 ; 20,22 ; 18,20)$ were judged as more acceptable by the Swedish than by the U.S. participants [mean difference $=$ $0.43 ; t(172)=4.0, p<.001]$. Pairs of odd multiples of 5 straddling a multiple of $10(15,25 ; 55,65)$ are judged as more acceptable by the U.S. than by the Swedish participants [mean difference $=0.64 ;[t(169)=4.1, p<.001]$.

Finally, consistent with the results from Experiments 1 and $2 \mathrm{~A}$ with regard to Question 3 from the introduction, the acceptability ratings of approximating pairs that conform to Pollmann and Jansen's (1996) rules were higher than they were for pairs that violated at least one of these rules [mean difference $=1.48 ; t(173)=21.0, p<.001$ ] An examination of individual pairs revealed one exception: The pair $(20,40)$, which conforms to the rules, is rated lower than the average of the 9 pairs that violate at least one rule [mean difference $=-0.20 ; t(169)=$ $-2.0, p<.05]$. The low-rated pair $(20,40)$ stands out as offering very low precision - that is, a very small ratio $(20 / 40=0.5)$ between its numbers. Recall the $5 \%$ rule of Pollmann and Jansen, which says that the precision must not be greater than 0.95; our present finding suggests that the precision must also not be too poor. We explored this idea further in Experiment 3.

\section{EXPERIMENT 3}

Our previous experiments revealed substantial consistency across Sweden and the U.S. in the usage, generation, and rated acceptability of approximating pairs of numbers used in estimation. For the most part, these were among the approximating pairs defined by Pollmann and Jansen's (1996) rules, but we have also seen patterns not covered by these rules, such as a preference for precision of the approximation (in Experiment 2B) and for using numbers that are small and numbers that are divisible by 5 (Experiment 2A). In our third experiment, we presented our participants with approximating pairs and asked them to judge the familiarity of each pair (familiarity being related to both usage and acceptability). We then used Pollmann 
Table 4

Mean Acceptability Judgments of Expressions Involving Approximating Pairs

\begin{tabular}{cccccccc}
\hline Pair & Judgment & $\begin{array}{c}\text { U.S. } \\
\text { Sample }\end{array}$ & $\begin{array}{c}\text { Swedish } \\
\text { Sample }\end{array}$ & Pair & Judgment & $\begin{array}{c}\text { U.S. } \\
\text { Sample }\end{array}$ & $\begin{array}{c}\text { Swedish } \\
\text { Sample }\end{array}$ \\
\hline$+20,25$ & 4.4 & 4.2 & 4.6 & $+50,60$ & 4.2 & 4.1 & 4.3 \\
$+20,30$ & 4.0 & 4.3 & 3.7 & $+55,60$ & 4.1 & 4.0 & 4.2 \\
$+18,20$ & 3.6 & 3.4 & 3.9 & 55,65 & 3.0 & 3.2 & 2.8 \\
$+20,22$ & 3.5 & 3.3 & 3.8 & 60,62 & 3.0 & 2.7 & 3.5 \\
20,23 & 2.8 & 2.6 & 3.2 & 58,60 & 2.7 & 2.5 & 2.9 \\
15,25 & 2.7 & 3.0 & 2.1 & 50,70 & 2.5 & 2.5 & 2.5 \\
$+20,40$ & 2.3 & 2.4 & 2.2 & 57,60 & 2.4 & 2.2 & 2.7 \\
18,22 & 2.0 & 2.0 & 2.1 & 58,62 & 1.8 & 1.8 & 1.8 \\
\hline
\end{tabular}

Note-The judgments were made on a scale from 1 (sounds completely unnatural) to 5 (sounds completely natural). A "+" signifies that the pair satisfies the four conditions of Pollmann and Jansen (1996). Standard deviations of judgments were all between 1.0 and 1.4.

and Jansen's rules, and the new rules we identified above, to predict familiarity ratings. From this analysis, we obtained more detailed answers to which rules were used (Question 3) and whether there was any difference in rules used in English and Swedish (Question 1). We also analyzed individual variation - that is, whether some people, independent of nationality, favored some rules more than other people favored the same rules (Question 4).

\section{Method}

A 23-item questionnaire was administered to the same 105 U.S. college students who had participated in Experiment 2 and to 94 Swedish retirees (older than 65) from the "pensioners' university" in Västerås, Sweden. The items were expressions of the form "10 or 13 years ago" for numbers between 2 and 40. Participants were asked to rate the familiarity of the expression on a 1 (never used) to 4 (often used) scale.

Eleven pairs conformed to Pollmann and Jansen's (1996) rules; the remaining 12 pairs each violated at least one of the rules. However, no violations of the "second number" rule were included, because the results of our previous experiments indicated that this rule is basically never violated. Thus, the second number rule was not assessed in Experiment 3.

Ratings were analyzed via multilevel modeling using PROC MIXED (SAS Institute Inc., 2004). This procedure allows for simultaneous analysis of cultural and individual differences in the relative weighting of one rule or another in making the familiarity judgments. In other words, the procedure allowed us to determine whether the U.S. or Swedish participants differed in how highly they weighted one rule over another, whether different individuals weighted rules differently, and whether an individual who weights one rule highly is significantly more or less likely to weight another rule highly.

Pollmann and Jansen's (1996) rules were dummy coded 1 for the rule being met, and 0 otherwise. To assess the feasibility of other rules for predicting the familiarity of approximating pairs, we generated three additional arithmetical rules on pairs $(a, b)$ :

The precision rule: More precise approximations are more acceptable. Precision is operationalized as the ratio between the smaller and larger number: $a / b$ (assuming that $a<b$, which holds for all pairs used in Experiment 3).

The smallness rule: Smaller numbers are more acceptable for use in approximating pairs. Smallness is operationalized as the inverse size of the average of the two numbers: $2 /(a+b)$.

The divisibility-by-5 rule: Both numbers in the pair must be divisible by 5 (dummy coded 1 for the rule being met, 0 otherwise).
The motivation for the precision rule is the finding in Experiment 2B that the very imprecise approximating pair $(20,40)$ was rated very poorly, despite satisfying all of the rules of Pollmann and Jansen. The smallness rule is motivated by the results of our Experiments 1 and 2A, as well as by Dehaene and Mehler's (1992) finding that smaller valued numbers in general occur more frequently than larger ones. Finally, the motivation for the divisibility-by-5 rule is the finding in Experiment 2A that numbers used in approximating pairs are typically divisible by 5 .

\section{Results}

Mean ratings are shown in Table 5. As in the previous experiment, pairs that conform to Pollmann and Jansen's (1996) four rules are rated highly, with a few exceptions $(20,22 ; 20,40)$. Table $6 \mathrm{~A}$ shows the results from a single multilevel model (with responses nested within raters and raters nested within countries), indicating which features of an approximating pair are judged as most familiar (main effects) and the degree of cross-cultural agreement on these features (rule $\times$ country interactions). The analysis provides an estimate of the relation between each of the corresponding rules and the rated familiarity of pairs, independent of the effects of the other rules included in the model (i.e., results are based on the Type III sums of squares). In the model, we also included random effects to assess individual differences (Table 6B).

Table 5

Means and Standard Deviations of Judgments of Familiarity of Approximating Pairs

\begin{tabular}{|c|c|c|c|c|c|}
\hline \multirow[b]{2}{*}{ Pair } & \multicolumn{2}{|c|}{ Judgment } & \multirow[b]{2}{*}{ Pair } & \multicolumn{2}{|c|}{ Judgment } \\
\hline & $M$ & $S D$ & & $M$ & $S D$ \\
\hline$+20,25$ & 3.5 & 0.7 & $+20,22$ & 2.2 & 1.0 \\
\hline$+10,15$ & 3.5 & 0.8 & 15,25 & 1.9 & 1.0 \\
\hline$+10,20$ & 3.4 & 0.9 & 2,5 & 1.8 & 1.1 \\
\hline$+20,30$ & 3.3 & 0.8 & 5,8 & 1.8 & 0.9 \\
\hline$+10,12$ & 3.1 & 0.8 & 20,23 & 1.8 & 0.8 \\
\hline$+8,10$ & 3.0 & 0.9 & 13,15 & 1.7 & 0.8 \\
\hline$+18,20$ & 2.7 & 0.9 & $+20,40$ & 1.7 & 1.0 \\
\hline$+2,4$ & 2.6 & 1.1 & 10,13 & 1.7 & 0.8 \\
\hline 12,15 & 2.5 & 0.9 & 12,16 & 1.4 & 0.6 \\
\hline 20,21 & 2.4 & 1.1 & 18,22 & 1.4 & 0.6 \\
\hline$+6,8$ & 2.4 & 1.0 & 13,18 & 1.3 & 0.6 \\
\hline 5,7 & 2.3 & 1.0 & & & \\
\hline
\end{tabular}

Note-Pairs were rated on a scale from 1 (never used) to 4 (often used). A " + " signifies that the pair satisfies the four conditions of Pollmann and Jansen (1996). 
Table 6A

Multilevel Model of Familiarity Ratings of the 23

Approximating Pairs From Experiment 3

\begin{tabular}{lrrrrr}
\hline \multirow{1}{*}{ Effect } & \multicolumn{5}{c}{ Fixed Effects } \\
\cline { 2 - 6 } \multicolumn{1}{c}{ Estimate } & \multicolumn{1}{c}{$S E$} & $d f$ & $t$ Value & $p r>|t|$ \\
\hline Intercept & 1.5082 & 0.07622 & 195 & 19.79 & $<.0001$ \\
Divisor rule & 0.6213 & 0.03242 & 195 & 19.16 & $<.0001$ \\
5\% rule & 0.3929 & 0.06380 & 3441 & 6.16 & $<.0001$ \\
Favorite numbers rule & 0.05009 & 0.03318 & 3441 & 1.51 & .1312 \\
Precision & 4.0987 & 0.1952 & 193 & 21.00 & $<.0001$ \\
Smallness & 7.6378 & 0.3415 & 195 & 22.36 & $<.0001$ \\
Divisibility-by-5 rule & 1.5597 & 0.05913 & 193 & 26.38 & $<.0001$ \\
Country & 0.3215 & 0.07144 & 3441 & 4.50 & $<.0001$ \\
Divisor $\times$ country & -0.2337 & 0.03039 & 3441 & -7.69 & $<.0001$ \\
5\% $\times$ country & -0.2448 & 0.05981 & 3441 & -4.09 & $<.0001$ \\
Favorite numbers $\times$ country & 0.06395 & 0.03110 & 3441 & 2.06 & .0398 \\
Precision $\times$ country & -0.2427 & 0.1829 & 3441 & -1.33 & .1848 \\
Smallness $\times$ country & 0.6589 & 0.3201 & 3441 & 2.06 & .0396 \\
Divisibility by $5 \times$ country & 0.1940 & 0.05542 & 3441 & 3.50 & .0005 \\
\hline
\end{tabular}

Note-For a pair $(a, b)$, the smallness variable is defined as $2 /(a+b)$, and the precision variable as $a / b$. Remaining rules are dummy coded ( 1 if the rule is met, 0 otherwise). Country is coded 1 for U.S. and $-s / u$ for Sweden, where $u$ is the number of U.S. observations, and $s$ is the number of Swedish observations, thus weighting countries equally in the analysis.

Table 6B

Variance and Covariance Parameter Estimates From the Multilevel Model

\begin{tabular}{lccccc}
\hline & 1 & 2 & 3 & 4 & 5 \\
\hline 1. Intercept & $0.214^{* * *}$ & & & & \\
2. Divisor & 0.026 & $0.044^{*}$ & & & \\
3. Precision & $0.264^{*}$ & $0.344^{* * *}$ & 0.602 & & \\
4. Smallness & 0.018 & 0.181 & -0.866 & 1.454 & \\
5. Divisibility by 5 & $-0.088^{*}$ & $0.101^{* * *}$ & $0.272^{* * *}$ & $-0.792^{* *}$ & see note \\
\hline
\end{tabular}

Note-The variance for factor 5 was not estimated by SAS. ${ }^{*} p<.05 .{ }^{* *} p<$ $.01 .{ }^{* * *} p<.001$.

Table 6A shows the importance of rules in judgments of familiarity. The interpretation of the unstandardized regression weights (in the column labeled "Estimate") is change in familiarity ratings associated with meeting a rule, keeping other rules the same. As an example, pairs that met Pollmann and Jansen's (1996) divisor rule are rated 0.62 higher on the $1-4$ scale than are pairs that do not satisfy this rule, independent of whether the pair is consistent with other rules listed in the table. Table 6A shows that, with the exception of the favorite numbers rule, all of the rules we tested were positively related to familiarity ratings. The magnitudes of the $t$ values in Table 6A provide an indication of the comparative importance of the different rules, independent of all other rules. As can be seen, of Pollmann and Jansen's three rules, only the divisor rule comes close to the magnitude of the $t$ values of the rules of precision, smallness, and divisibility by 5 .

We now turn to the question of whether rules apply equally in the U.S. and Sweden. Significant interactions of rules and country in Table 6A indicate that the relative importance of the corresponding rule varied between the U.S. and Swedish participants; the sign of the coefficient indicates whether a rule was relied on more (positive sign) or less (negative sign) heavily by U.S. than by Swedish participants. The table shows that the Swedish participants weighted the divisor and 5\% rules more heavily than did the U.S. participants. In contrast, the U.S. participants weighted the rules of favorite numbers, smallness, and, in particular, divisibility by 5 more heavily; the latter is in line with our finding in Experiment $2 \mathrm{~B}$ that divisibility by 5 is more favored by Americans.

Finally, we turn to the question of individual variation. For this analysis, we examined the four rules with the largest $t$ values in Table 6A: the divisor, precision, smallness, and divisibility-by-5 rules. Table $6 \mathrm{~B}$ shows variances and covariances for individuals' weightings of these rules. The divisor rule (which arguably is the most cognitively demanding of all proposed rules) shows significantly high variance, indicating substantial individual differences in the reliance on this rule. A high covariance in Table $6 \mathrm{~B}$ indicates that individuals' reliance on one rule is substantially related to their reliance on another rule. We see that individuals who rely on the divisor rule also tend to rely on the divisibility-by-5 and precision rules; in contrast, the divisibility-by-5 rule is negatively related to the smallness and precision rules.

\section{Discussion}

In Experiment 3, we addressed Questions 3 and 4, specifically, to assess which set of rules best captures the fa- 
miliarity of approximating pairs in Swedish and English, and whether there are individual differences in the implicit use of these rules in making familiarity judgments.

Motivated by our findings in previous studies, we generated a collection of new rules (smallness, precision, and divisibility by 5). We found that these new rules were of greater importance than the rules of Pollmann and Jansen (1996).

The same set of rules applied in both the Swedish and American samples, but there were country differences in the extent to which participants weighted some rules versus others in the familiarity rating. For instance, Swedish participants gave more weight to the divisor rule, and less weight to the divisibility-by-5 rule, than did American participants. This is consistent with our findings in Experiment $2 \mathrm{~B}$, supporting that this difference is country based (rather than age based; recall that the samples consisted of U.S. college students and Swedish retirees).

We found evidence for significant individual differences in the extent to which individuals rely on the divisor rule, and that individuals who rely heavily on this rule also tend to rely on the precision and divisibility-by-5 rules.

\section{EXPERIMENT 4}

Finally, we turn to the fifth, and last, question on our list: Do the rules for approximating pairs depend on the unit of measurement?

\section{Method}

A questionnaire was administered to 45 Swedish college students from Mälardalen University. The questionnaire contained 15 expressions, such as " 10,15 days ago" and " 12,18 hours ago." The 15 expressions were created by a combination of three units of time with different bases $(7,24,60$; days, hours, minutes, respectively), with five approximating pairs that were chosen to fit better with one of the units than with the others: $(14,21)$ fits with base 7 (days); $(12,18)$ and $(8,12)$ fit with base 24 (hours); and $(10,15)$ and $(30$, 45) fit with base 60 (minutes). As in Experiment 2B, participants were asked to rate how natural they thought the expression would sound in speech, on a 1 (completely unnatural) to 5 (completely natural) scale.

\section{Results and Discussion}

Mean ratings are shown in Table 7. For each approximating pair, acceptability is highest for the predicted case (although not significantly so in one case). This indicates

Table 7

Mean Ratings of Acceptability for the 15 Combinations of Approximating Pairs and Units From Experiment 4

\begin{tabular}{cccc}
\hline Pair & Hours & Minutes & Days \\
\hline $10,15^{*}$ & 3.8 & 4.4 & 4.0 \\
$14,21^{*}$ & 1.6 & 1.8 & 2.3 \\
$12,18^{*}$ & 2.7 & 2.4 & 2.3 \\
8,12 & 3.3 & 3.0 & 3.0 \\
$30,45^{* * *}$ & 2.0 & 3.4 & 2.3 \\
\hline
\end{tabular}

Note-For each pair, the highest rating is in italics. Asterisks indicate the statistical significance of the difference between the highest and second-highest rating. Standard deviations were all below 1.5. ${ }^{*} p<$ $.05{ }^{* * *} p<.001$. that the grammar of approximating pairs is sensitive to context-specifically, to the often used bases associated with hours, minutes, and days.

\section{GENERAL DISCUSSION}

We begin by summarizing our answers to the research questions listed in the introduction, and then we draw some general conclusions about the nature of the "grammar of approximating pairs."

First, are the same approximating pairs used, and found acceptable, in Swedish and English? On the whole, the same patterns that were identified in the Dutch corpora also emerged in both the Swedish and English corpora, and they were confirmed in Experiments 2A and 2B, in which both usage and acceptability were assessed through questionnaires. There indeed seems to exist an important regularity across languages in the usage of pairs of numbers to estimate a quantitative range. However, within the same set of rules, there seem to exist national differences in the relative weighting of these rules (Experiments 2B and 3).

Second, is the acceptability of approximating pairs dichotomous, as was implicitly assumed by Pollmann and Jansen (1996)? Our participants used the entire 5-point scale of acceptability in Experiment 2B, clearly indicating that they do not cognitively treat approximating pairs as either wholly acceptable or wholly unacceptable. This helps to explain the usage patterns we found in Table 1, in which approximating pairs used in colloquial speech show an extremely large variation in how often they are used.

Third, to what extent does Swedish and English usage of approximating pairs follow the rules proposed by Pollmann and Jansen (1996), and are there additional rules? Both our corpora analysis and questionnaires indicate that the rules proposed by Pollmann and Jansen are quite good at predicting usage and acceptability of approximating pairs that are used to estimate a quantitative range. However, Experiments $2 \mathrm{~A}$ and $2 \mathrm{~B}$ revealed some exceptions. First, the inclusion of 2.5 in the set of "favorite numbers" seems entirely unwarranted. Second, we found an unpredicted lack of acceptance for very imprecise approximating pairs. Third, in addition to Pollmann and Jansen's rules about the difference, there seem to exist preferences for certain features (smallness and divisibility by 5) of the individual numbers. Indeed, the results for Experiment 3 revealed the precision, smallness, and divisibility-by-5 rules to be even more important than the rules from Pollmann and Jansen.

Fourth, are there individual differences in the application of these rules? As an illustration of individual variation, in Experiment $2 \mathrm{~B}$, the pair $(60,62)$ was given the highest possible acceptability judgment (5) by more than $20 \%$ of the participants, whereas almost the same proportion gave it the lowest possible judgment (1). The results of Experiment 3 confirmed that there is substantial individual variation in the reliance on the divisor rule, and covariation with the reliance on the precision and divisibility-by-5 rules, when making judgments about the familiarity of approximating pairs. 
Fifth, are the rules for the usage of approximating pairs context sensitive? Yes. In Experiment 4, we were able to manipulate the acceptability ratings of number pairs by varying the units within which they were presented (" 30 , 45 minutes" vs. " 30,45 hours"). Pairs that conformed to the base represented in the context (e.g., minutes, weeks) were judged as more acceptable than pairs that did not conform.

To summarize, the same patterns of usage of approximating pairs to estimate a quantitative range are found in several language corpora (Dutch, Swedish, and American) and across questionnaire studies of American students, Swedish students, and Swedish retirees. The consistency suggests that there is an underlying cognitive basis - the consistency cannot be simply due to social influences - that is common across languages (and apparently across generations). This is in keeping with Dehaene and Mehler's (1992) interpretation regarding the regularities in the distribution of single numbers across languages and cultures. Our findings on context sensitivity in Experiment 4 suggest that people's set of acceptable approximate ranges for estimating quantity depends on the underlying base of the pair being estimated. Knowledge of the bases themselves (e.g., base 10, base 60) cannot be inherent, but their common usage appears to at least implicitly result in the development of anchoring numerals for representing and communicating estimated ranges that reflect regularity in these base systems. For instance, the anchors of 5,10 , and 15 in the context of minutes are all divisors of the base 60 .

Two general rules - smallness and precision-emerged as complements to Pollmann and Jansen's (1996) rules. As was reported by Dehaene and Mehler (1992), small numbers are more common than large numbers in many modern contexts, and the underlying representations for small numbers are more accurate. The latter means that differences among small numbers are more meaningful in terms of conveying a potential range of values than are the differences among larger numbers. Thus, the smallness rule may stem from approximating pairs that include small numbers being disproportionately efficient in communicating a quantitative range.

We note that all of our findings and those of Pollmann and Jansen (1996) and Dehaene and Mehler (1992) are based on samples drawn from modern, educated countries. It is almost certain that exposure to modern mathematical concepts in school, such as the base-10 system, and other modern tools (e.g., clocks) influenced our results (Geary, 2007). At the same time, data from all of these studies suggest that a personal "grammar" for generating number pairs for estimating a range of quantities exists, and that people may be more or less sensitive to this grammar. These "grammatical rules" do not appear to be of the complexity associated with language, but there are nonetheless regularities in how people parse the magnitudes associated with the potentially inherent system that supports approximate representations of magnitude. At the very least, our findings and those of Pollmann and Jansen suggest that people not only implicitly develop anchor numerals for accessing relative value represented by the approximate magnitude system, as was found by Dehaene and Mehler, but also pairs of anchors for estimating and communicating quantitative range.

Finally, there are many questions left unanswered and thus many directions for future research. The analogy with grammatical acceptability suggests that questions and methods may carry over from that school of research. For instance, it has been shown that grammatical acceptability of expressions is sensitive to mere exposure to similar expressions (Luka \& Barsalou, 2005). How many exposures to approximating pairs in the context of colloquial speech are needed for people to generate pairs of estimation anchors? Does the ease of the generation of these anchors vary with age, and can colloquial anchor pairs be used to facilitate children's mathematical learning, as in the teaching of the meaning of line segments on the mathematical number line? Second, investigations into the rather large individual variation we found in rules about approximating expressions might be informed by psycholinguistic research on individual variation in grammatical intuitions (Gordon \& Hendrick, 1998). In particular, one study explained individual variation in ratings of a certain grammatical construction by differences in how individuals perceived the constituent structure of the sentences, as was measured by RTs to a target word in a certain grammatical position (Gerken \& Bever, 1986). In the same vein, one might speculate whether variation in intuitions about approximations is somehow related to differences in perceptions of numbers. Finally, approximating pairs have so far been studied only in Germanic languages; are the same patterns present in all language groups?

\section{AUTHOR NOTE}

K.E. acknowledges support from the CULTAPTATION project (European Commission Contract FP6-2004-NEST-PATH-043434), and D.C.G. acknowledges support from Grant R37 HD045914 from the National Institute of Child Health and Human Development. We are grateful to Mark Ashcraft for many valuable comments. Address correspondence to K. Eriksson, School of Education, Culture and Communication, Mälardalen University, Box 883, SE-72123, Västerås, Sweden (e-mail: kimmo.eriksson@mdh.se).

\section{REFERENCES}

Chagnon, N. A. (1997). Yanomamö (5th ed.). Fort Worth, TX: Harcourt.

Channel, J. (1980). More on approximations: A reply to Wachtel. Journal of Pragmatics, 4, 461-476.

Chomsky, N. (1981). Lectures on government and binding. Dordrecht: Foris.

Davies, M. (2008). The Corpus of Contemporary American English (COCA): 385 million words, 1990-present. Available at www .americancorpus.org.

Dehaene, S. (1992). Varieties of numerical abilities. Cognition, 44, $1-42$.

Dehaene, S. (1997). The number sense: How the mind creates mathematics. New York: Oxford University Press.

Dehaene, S., \& Mehler, J. (1992). Cross-linguistic regularities in the frequency of number words. Cognition, 43, 1-29.

Fuson, K. C. (1988). Children's counting and concepts of number. New York: Springer.

Gallistel, C. R., \& Gelman, R. (1992). Preverbal and verbal counting and computation. Cognition, 44, 43-74.

Geary, D. C. (2006). Development of mathematical understanding. In 
W. Damon, R. M. Lerner, D. Kuhn, \& R. S. Siegler (Eds.), Handbook of Child Psychology: Vol. 2. Cognition, perception, and language (pp. 777-810). New York: Wiley.

Geary, D. C. (2007). Educating the evolved mind: Conceptual foundations for an evolutionary educational psychology. In J. S. Carlson \& J. R. Levin (Eds.), Educating the evolved mind: Vol. 2. Psychological perspectives on contemporary educational issues (pp. 1-99). Greenwich, CT: Information Age.

GERKEN, L., \& BEVER, T. G. (1986). Linguistic intuitions are the result of interactions between perceptual processes and linguistic universals. Cognitive Science, 10, 457-476.

Gordon, P. C., \& HendRICK, R. (1998). Dimensions of grammatical coreference. In M. A. Gernsbacher \& S. J. Derry (Eds.), Proceedings of the Twentieth Annual Conference of the Cognitive Science Society (pp. 424-429). Hillsdale, NJ: Erlbaum.

LANGENDOen, D. T. (2006). Disjunctive numerals of estimation. Style, 40, 46-55.

Luka, B. J., \& Barsalou, L. W. (2005). Structural facilitation: Mere exposure effects for grammatical acceptability as evidence for syntactic priming in comprehension. Journal of Memory \& Language, $\mathbf{5 2}, 436-459$.
Pica, P., Lemer, C., Izard, V., \& Dehaene, S. (2004). Exact and approximate arithmetic in an Amazonian indigene group. Science, 306, 499-503.

Pollmann, T., \& Jansen, C. (1996). The language user as an arithmetician. Cognition, 59, 219-237.

SAS Institute INC. (2004). Statistical analysis system 7.5 [Computer software]. Cary, NC: Author.

SAXE, G. B. (1981). Body parts as numerals: A developmental analysis of numeration among the Oksapmin of Papua New Guinea. Child Development, 52, 306-316.

Siegler, R. S., \& Opfer, J. (2003). The development of numerical estimation: Evidence for multiple representations of numerical quantity. Psychological Science, 14, 237-243.

Sigurd, B. (1988). Round numbers. Language in Society, 17, 243-252.

Spelke, E. S. (2000). Core knowledge. American Psychologist, 55, 1233-1243.

The Swedish PAROLE CORPus. Institutionen för svenska språket vid Göteborgs Universitet. Available at http://spraakbanken.gu.se/parole/.

(Manuscript received May 22, 2009;

revision accepted for publication September 28, 2009.) 